\title{
Comparison of Penetration Depth and Microleakage of Resin Infiltrant and Conventional Sealant in Pits and Fissures of Permanent Teeth In Vitro
}

\author{
Amani Ahmad Al Tuwirqi ${ }^{1}$, Ahmad Mohammad Alshammari ${ }^{2}$, Osama Mahmood Felemban ${ }^{3}$, Najat M Ali Farsi ${ }^{4}$
}

\begin{abstract}
Aim: To assess the penetration and microleakage of a resin infiltrant for the prevention of noncavitated white lesion progression in the fissure system of permanent teeth and compare it to a conventional sealant.

Materials and methods: Sixty extracted permanent teeth with artificially produced noncavitated white lesions were randomly distributed according to the restorative material into group I (experimental) which received ICON resin infiltration, and group II (control) which received Helioseal F fissure sealant. For the penetration test, restorative materials were mixed with a fluorescent dye (eosin $\mathrm{H}$ ) before light curing; roots were then amputated, and three buccopalatal microscopic sections were obtained from each tooth. These were dipped in $0.001 \mathrm{mg}$ of ethanolic sodium fluorescence solution. Material penetration depth was assessed through confocal microscopy. Thirty teeth (15 from each group) underwent a microleakage test. Teeth were thermocycled, and then immersed in $50 \%$ silver nitrate $\left(\mathrm{AgNO}_{3}\right)$ solution for 3 hours. Microleakage was evaluated using X-ray microtomography (micro-CT); $\mathrm{AgNO}_{3}$ presence between the material and the tooth surface indicated microleakage. Results: The mean material penetration value in teeth treated with ICON $(104.8571 \pm 7.63360 \mu \mathrm{m})$ was significantly higher than that in teeth treated with Helioseal F $(5.3158 \pm 3.83825 \mu \mathrm{m})(p<0.001)$. The mean microleakage value in demineralized enamel treated with Helioseal $\mathrm{F}$ $(0.2238 \pm 0.12561)$ was significantly greater than that in demineralized enamel treated with ICON $(0.0119 \pm 0.0097)(p<0.001)$.

Conclusion: ICON displayed more penetration and less microleakage than Helioseal F in pits and fissures of permanent teeth.

Clinical significance: Resin infiltrant showed superior retention and can provide a good alternative for adhesives and fissure sealants.

Keywords: Fissure sealant, ICON, Microleakage, Penetration.

The Journal of Contemporary Dental Practice (2019): 10.5005/jp-journals-10024-2715
\end{abstract}

\section{INTRODUCTION}

Despite major advances in preventive dentistry, dental caries is considered the most common chronic disease; they are reported more often in American children than asthma or allergic rhinitis. ${ }^{1,2}$ In Kingdom of Saudi Arabia, it a public-health problem affecting children at different ages. 3,4

Protocols for the prevention of dental caries have improved in recent decades. As a result, the occurrence of dental caries on the smooth and proximal surfaces of teeth has decreased dramatically. However, the occurrence of occlusal caries (in pits and fissures) was not much affected by the preventive protocols; their prevalence actually increased. ${ }^{5}$

Sealants can prevent or slowdown the progression of caries by acting as a barrier between the tooth fissure system and the oral environment, food debris, and acid-producing microorganisms. ${ }^{6,7}$ Reports indicate that sealants effectively prevent tooth decay; however their retention is questionable. ${ }^{8,9}$ Micro-mechanical retention is crucial for sealants to prevent dental caries. Therefore, if any part of a sealant is dislodged from its place, the preventive abilities of the material are compromised. Additionally, microleakage around the sealant reduces its effectiveness.

Resin infiltrant has commonly been used to treat initial proximal caries. More recently, it was introduced as a fissure sealant. Few studies have compared its effectiveness for the prevention of dental caries to that of sealant material when used in pits and fissures. ${ }^{9-11}$ One of these studies reported that resin infiltrate had superior penetration into noncavitated carious fissures when compared to resin-based fissure sealant. ${ }^{10}$ Another study evaluated
1,3,4 Department of Pediatric Dentistry, King Abdulaziz University, Jeddah, Kingdom of Saudi Arabia

${ }^{2}$ Department of Pediatric Dentistry, Ministry of Health, Hafer Al-batin, Eastern Province, Kingdom of Saudi Arabia

Corresponding Author: Amani Ahmad Al Tuwirqi, Department of Pediatric Dentistry, King Abdulaziz University, Jeddah, Kingdom of Saudi Arabia, Phone: +966-540606742, e-mail: aaltuwirqi@kau.edu.sa

How to cite this article: Al-Tuwirqi AA, Alshammari AM, Felemban OM, et al. Comparison of Penetration Depth and Microleakage of Resin Infiltrant and Conventional Sealant in Pits and Fissures of Permanent Teeth In Vitro. J Contemp Dent Pract 2019;20(11):1339-1344.

Source of support: Nil

Conflict of interest: None

the nanoleakage of different sealing therapies when applied to noncavitated artificial enamel lesions on the labial surface of teeth and concluded that ICON can be used as a replacement for adhesives and fissure sealants. ${ }^{11}$

The aim of this study was to compare the depth of penetration and microleakage of a resin infiltrant and a conventional sealant when used in the fissures of permanent human teeth.

\section{Materials and Methods}

\section{Ethical Approval}

Ethical approval was obtained from the Research Ethics Committee, Faculty of Dentistry, KAU, Jeddah, Kingdom of Saudi Arabia (Approval no. 002-04-17).

(-) The Author(s). 2019 Open Access This article is distributed under the terms of the Creative Commons Attribution 4.0International License (https://creativecommons. org/licenses/by-nc/4.0/), which permits unrestricted use, distribution, and non-commercial reproduction in any medium, provided you give appropriate credit to the original author(s) and the source, provide a link to the Creative Commons license, and indicate if changes were made. The Creative Commons Public Domain Dedication waiver (http://creativecommons.org/publicdomain/zero/1.0/) applies to the data made available in this article, unless otherwise stated. 


\section{Research Design}

An experimental in vitro study was done (Flowchart 1).

\section{Study Sample}

Sixty extracted human premolars, collected from the departments of Oral Maxillofacial Surgery and Orthodontics in the College of Dentistry at King Abdulaziz University (KAU), were used in this experimental study. The selected teeth were extracted due to orthodontic reasons.

Only sound, nonstained, nonfractured, nonrestored, permanent teeth without developmental anomalies were included in the study. The sample size was calculated using G-power software program (V 3.1).

\section{Grouping}

Teeth were randomly distributed according to the restorative material into two main groups (I and II). Each group was further subdivided into two equal subgroups according to the in vitro test used.

Group I (experimental group) included 30 teeth treated with ICON resin infiltration (DMG, Chemisch-pharma, Fabrik GmbH, Hamburg, Germany).

- Group IA included 15 teeth, which underwent the penetration test.

- Group IB included 15 teeth, which underwent the microleakage test.Group II (control group) included 30 teeth treated with Helioseal F fissure sealant (Ivoclar Vivadent AG, Liechtenstein).

- Group IIA included 15 teeth, which underwent the penetration test.

- Group IIB included 15 teeth, which underwent the microleakage test.

Flowchart 1: Study design

\section{Sample Preparation}

The teeth were cleansed thoroughly with a rotating prophylaxis brush and a slurry of pumice to clear away any residual biofilm. The teeth were then kept in Thymol storage media (Sigma-Aldrich, Steinheim, Germany) until the start of the experiment. Teeth apices were closed with sticky wax to prevent dye penetration. Nail varnish was then applied all around the tooth, leaving a $1 \mathrm{~mm}$ margin away from the sealant. Each specimen was immersed individually for 1 to 2 weeks in separate plastic containers containing $10 \mathrm{~mL}$ of demineralizing solution, which induced lesions similar to those created by dental caries. ${ }^{12}$ The containers were color-coded and numbered from 1 to 60 . The teeth were evaluated visually using International Caries Detection and Assessment System (ICDAS) codes. ${ }^{13}$ Only teeth assessed as code 2 (distinct visual change in enamel) were included.

\section{Penetration-depth Test Procedure}

For the penetration test, teeth were assigned to group IA and group IIA. Group IA teeth, 15 teeth from the resin-infiltration group, were etched with $15 \%$ hydrochloric gel for 120 seconds following the manufacturer's manual. Group IIA teeth, 15 teeth from the fissuresealant group, were etched for 60 seconds with $37 \%$ phosphoric acid. Then teeth were air-dried for 10 seconds. Next, all specimens received their assigned sealing material mixed with $0.1 \%$ eosin $\mathrm{H}$ dye (Sigma-Aldrich, Steinheim, Germany); the materials were applied in the fissures of the occlusal surface. Finally, the materials were cured with light for 60 seconds.

The roots of the teeth were amputated, and the crowns of the specimens were mounted on an isomet holder. Three slices approximately 1,200 $\mu \mathrm{M}$ in thickness were obtained from different sites of the fissure after cutting the teeth in a buccolingual perpendicular direction using a diamond-metal bonded

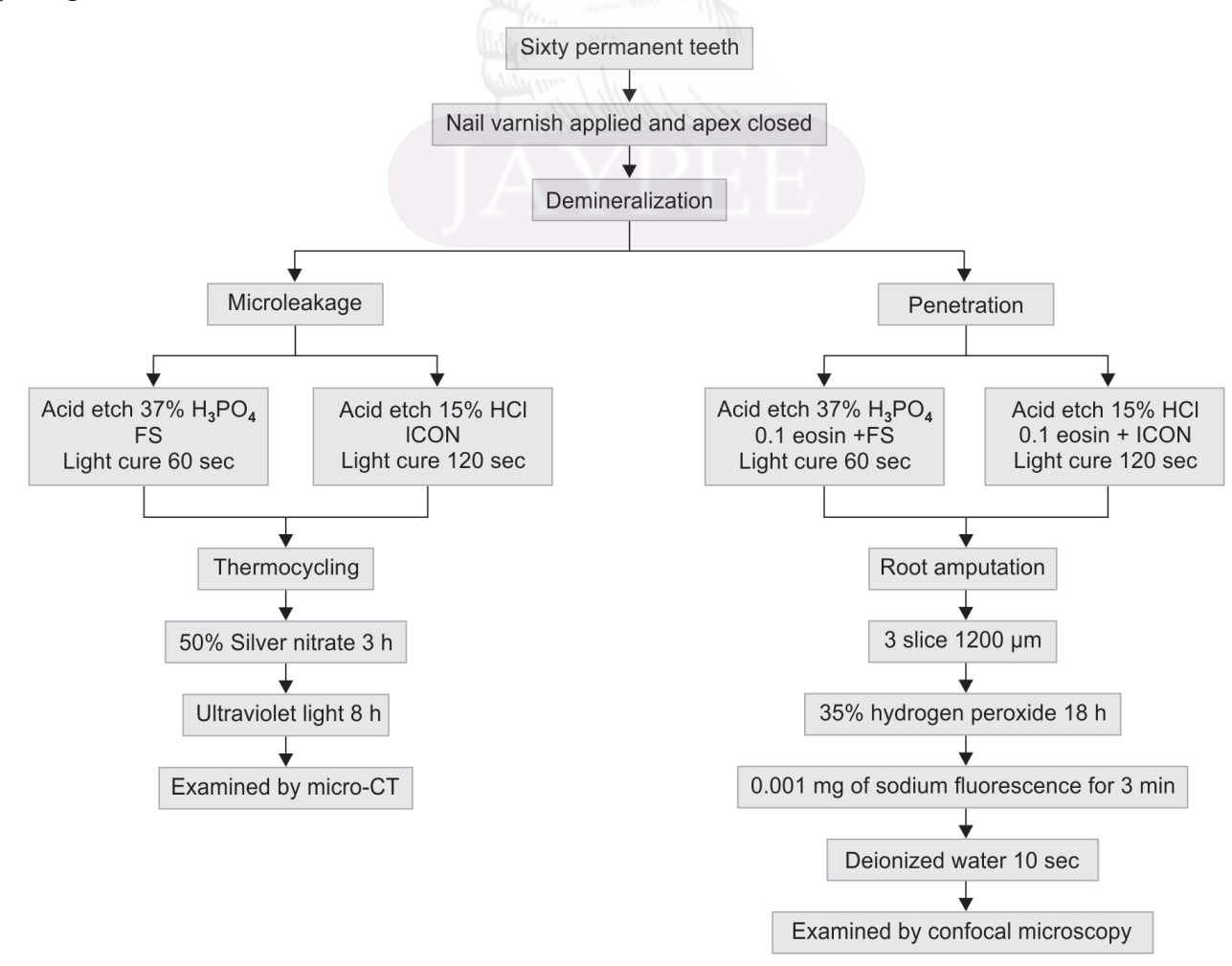


low-speed saw (Ted Pella, Inc., USA). Tooth sections were immersed in $35 \%$ hydrogen peroxide for 18 hours to remove all unattached fluorophore dye. The sections were dipped in a $50 \%$ ethanolic solution of $0.01 \mathrm{mg}$ sodium fluorescein (NaFl; Sigma-Aldrich, Steinheim) for 3 minutes to stain the nonpenetrated porous structures of the enamel. Finally, all of the specimens were washed for 10 seconds with deionized water before examination using confocal microscopy (Leica Microsystems GmbH, Wetzlar Germany). ${ }^{14}$

Each specimen was examined under a $10 \times$ magnification dual laser confocal microscope (Fig. 1A). Pictures were recorded and analyzed using LasX (Leica Microsystems GmbH, Wetzlar Germany). The penetration depth was measured in micrometers. Nine penetration points were recorded and the mean value measured for each microscopic slide.

\section{Microleakage Test}

Teeth were allocated to either group IB or group IIB. In group IB, the resin-infiltration group, 15 teeth were etched with $15 \%$ hydrochloric gel for 120 seconds following the manufacturer's manual. In group IIB, the fissure sealant group, 15 teeth were etched for 60 seconds with $37 \%$ phosphoric acid. Subsequently, the materials were applied in the fissures of the occlusal surfaces of the teeth at a level between the fissure and the tip of the cusp. The materials were then cured with light for 60 seconds. The teeth were then thermocycled (SD Mechatronik $\mathrm{GmbH}$, Germany) for 10,000 cycles between $5^{\circ} \mathrm{C}$ and $55^{\circ} \mathrm{C}$ water paths, for 30 seconds dwell.

The crowns of all teeth were stored in $50 \% \mathrm{AgNO}_{3}$ solution (Sigma-Aldrich Co., St. Louis, Mo) for 3 hours at $37^{\circ} \mathrm{C}$ in a dark container. The teeth were washed with deionized water, then subjected to ultraviolet light for 8 hours. To obtain the images, each of the 30 teeth was mounted on the specimen-holder of the X-ray microtomography system (model 1174, Skyscan Kontich, Belgium) (Fig. 1B). To scan all specimens, we used a 100-kV/98 mA X-ray source and a $1-\mathrm{mm}$ aluminum filter. At $180^{\circ}$, each specimen was rotated with a rotation step of $0.40^{\circ}$. The gain was set at 1.0 , and the exposure time was 3.7 seconds. Cone-beam reconstruction was applied to the projected images using NRecon (Skyscan Kontich, Belgium) (V1.6). We used CT-Analyzer (Skyscan Kontich, Belgium) (V.1.11) to obtain the total volume of our interest area, which was $1 \mathrm{~mm}$ from the outline of the material, and the total volume of the $\mathrm{AgNO}_{3}$, which represented the microleakage that occurred.
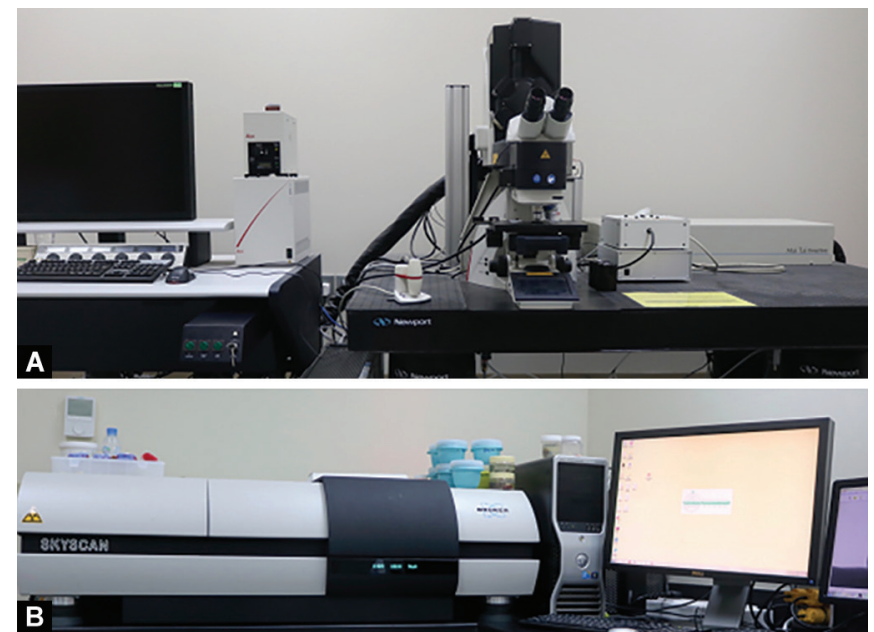

Figs 1A and B: (A) Confocal microscope (Leica Microsystems GmbH, Wetzlar Germany); (B) Micro-CT (model 1174, Skyscan Kontich, Belgium)

\section{Statistical Analysis}

Data were statistically analyzed using the Statistical Package for Social Sciences (SPSS) version 20.0 (SPSS Inc., Chicago, IL). The Mann-Whitney $U$ test was used to investigate the differences in penetration depth and microleakage. A $5 \%$ level of significance was used for all tests.

\section{Results}

\section{Penetration Depth Result}

Nine sections were destroyed during tooth sectioning: 3 were destroyed from the ICON group and 6 from the Helioseal F group. The remaining 81 sections were examined, and the results revealed that the mean penetration depth of ICON was $104.8571 \pm 7.63360$ (Table 1). This was significantly greater than the mean penetration depth of Helioseal F, which was $5.3158 \pm 3.83825(p<0.001)$. Figures 2 and 3 illustrate the penetration depth, through porous enamel, of ICON and Helioseal F, respectively.

\section{Microleakage Result}

A total of thirty teeth were examined using X-ray microtomography, and the pictures were analyzed (Fig. 4). A ratio of the radio-opaque $\mathrm{AgNO}_{3}$ volume, which leaked between the tooth and the sealing material, over the total volume of restorative material used was calculated separately for each tooth. The ICON group showed smaller mean-ratio values of microleakage $(0.0119 \pm 0.0097)$ than the Helioseal F group $(0.2238 \pm 0.12561)$ (Table 2$)$. The difference was statically significant $(p<0.001)$.

\section{Discussion}

Penetration depth and microleakage tests provide valuable information to evaluate the infiltration ability of restorative materials. The aim of this laboratory study was to assess the penetration depth and microleakage of infiltration resin (ICON) in noncavitated carious fissures of human teeth and compare them to those of fissure sealant (Helioseal F). Our study revealed significantly better results with ICON than with Helioseal F. As retention of pit and fissure sealant poses a challenge in preventing dental caries; 8 resin infiltrant showed superior retention and can provide a good alternative for adhesives and fissure sealants.

Occlusal surfaces represent merely $12 \%$ of the entire exterior surface of teeth; despite this, they are eight times more susceptible to dental caries than other surfaces. ${ }^{15}$ The effectiveness of fissure sealant has been proven over time. ${ }^{16,17}$ However, the development of improved materials would allow for more effective prevention of dental caries.

Premolars, extracted for orthodontic reasons, were selected for this study because they do not differ significantly from one to another in terms of physical properties or chemical composition. ${ }^{18,19}$ We assumed this would reduce our sample variability. The process for enamel lesion formation was also designed to reduce variability among the samples, as all of the teeth were subjected to the same conditions.

\section{Penetration Depth}

Our results showed that ICON has a greater penetration depth than Helioseal F. This can be explained by the higher penetration coefficient of ICON, whereas a low penetration coefficient has been reported for Helioseal F. The viscosity, surface tension, and contact angle of the material in relation to the solid determines the penetration 
Table 1: Comparison of the mean penetration depth of ICON and Helioseal F

\begin{tabular}{|c|c|c|c|c|c|c|}
\hline & Material & $n$ & Mean & Standard deviation & Median & pvalue \\
\hline \multirow[t]{2}{*}{ Penetration depth } & ICON & 42 & 104.8571 & 7.63360 & 106 & $<0.001$ \\
\hline & Helioseal F & 38 & 5.3158 & 3.83825 & 6 & \\
\hline
\end{tabular}

\#Mann-Whitney $U$ test
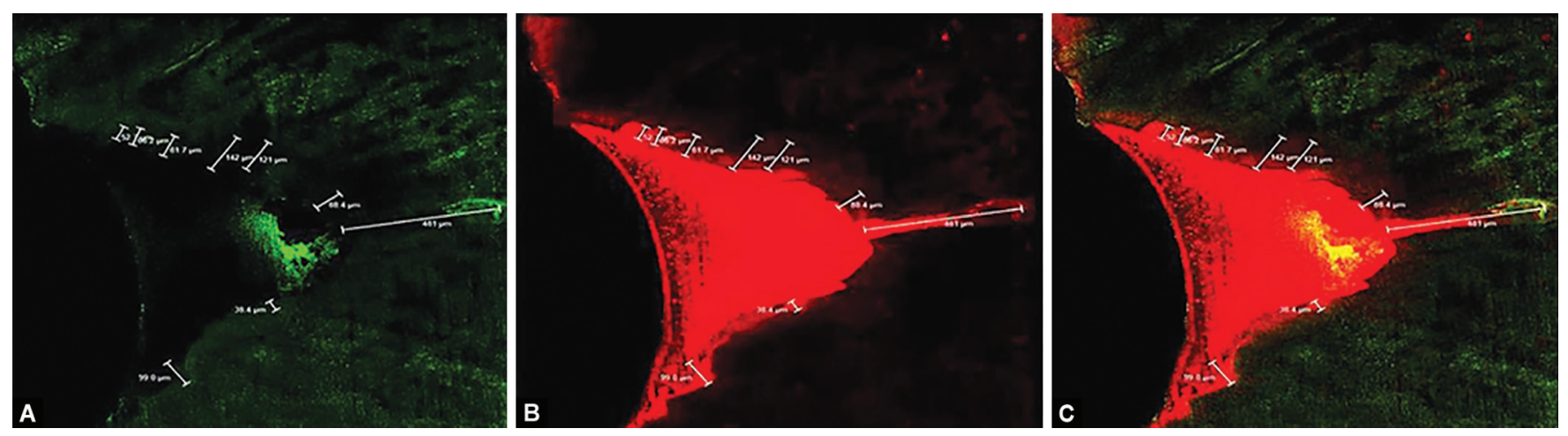

Figs $2 \mathrm{~A}$ to C: ICON penetration. (A) Porous enamel dyed with NaFI (green); (B) Penetration of ICON dyed with eosin $\mathrm{H}$ (red) into the enamel; (C) Penetration of ICON (red) into the porous enamel lesion (green)
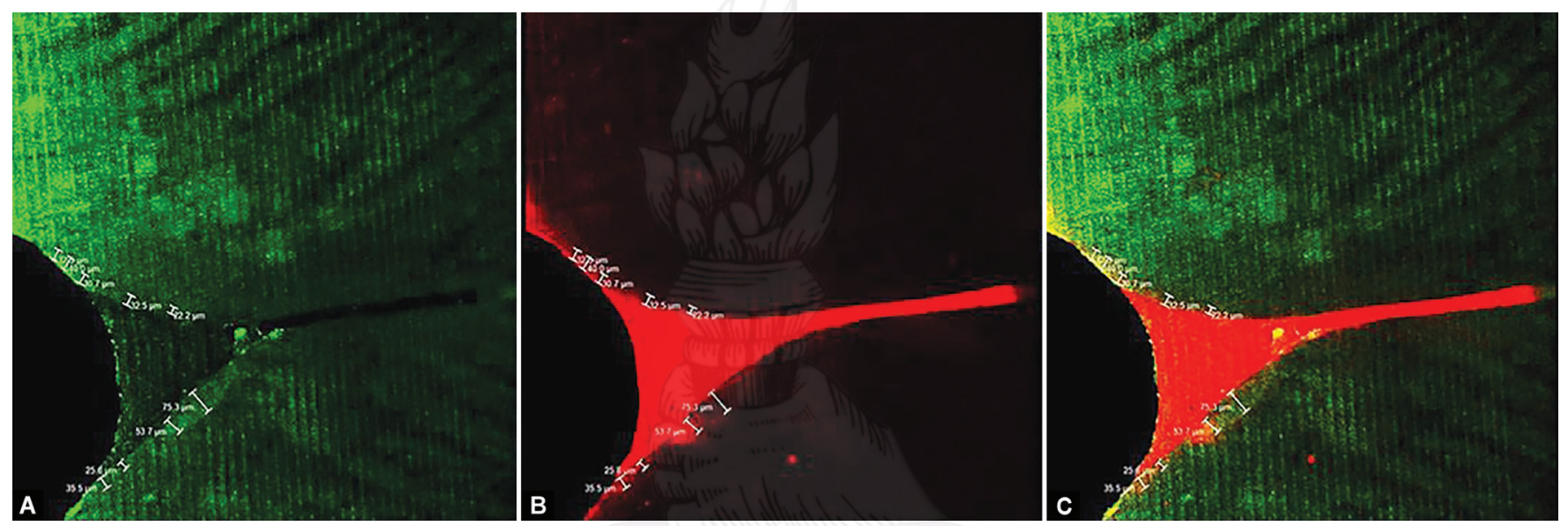

Figs 3A to C: Helioseal F penetration. (A) Porous enamel dyed with NaFI (green); (B) Penetration of Helioseal F dyed with eosin $\mathrm{H}$ (red) into the enamel; (C) Penetration of Helioseal $F($ red) into the porous enamel lesion (green)

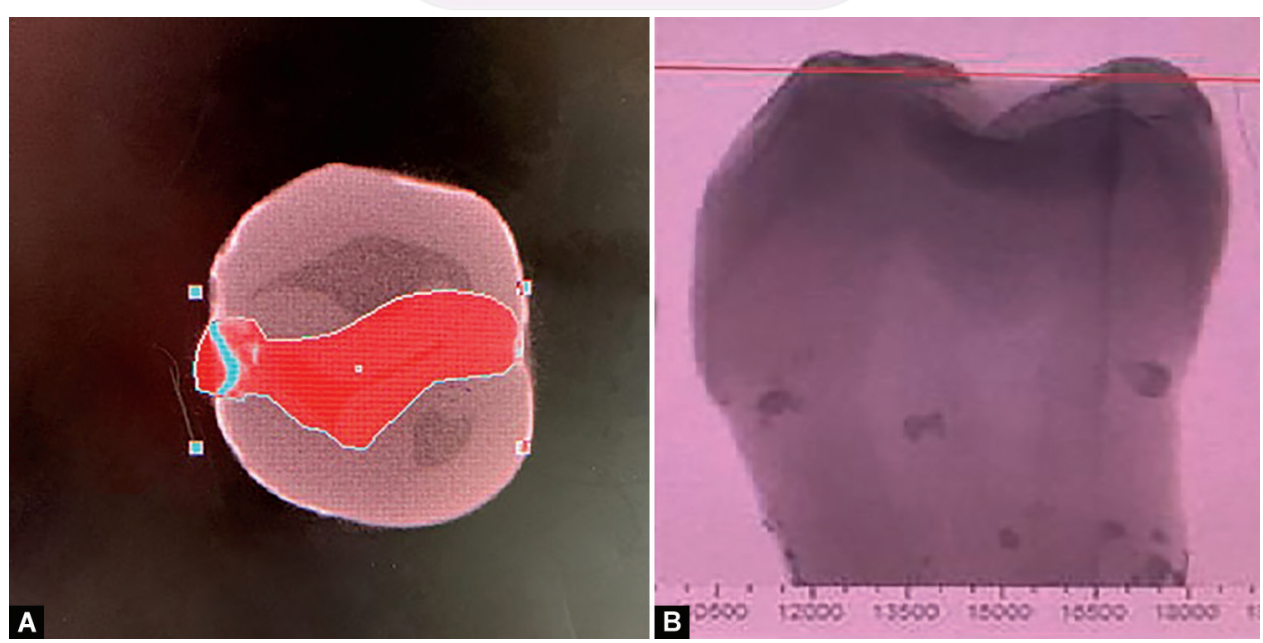

Figs 4A and B: Area of interest shown using cone-beam reconstruction with NRecon (Skyscan Kontich, Belgium) 
Table 2: Comparison of mean microleakage of ICON and Helioseal F

\begin{tabular}{|c|c|c|c|c|c|c|}
\hline & Material & $n$ & Mean & Standard deviation & Median & pvalue \\
\hline \multirow[t]{2}{*}{ Microleakage } & ICON & 15 & 0.0119 & 0.00970 & 0.0118 & $<0.001$ \\
\hline & Helioseal F & 15 & 0.2238 & 0.12561 & 0.223 & \\
\hline
\end{tabular}

\#Mann-Whitney U test

coefficient. ${ }^{20}$ In addition, ICON preparation contains mainly triethylene-glycol-dimethacrylate (TEGDMA) as demonstrated by Sideridou et al., ${ }^{21}$ resins that contain mostly TEGDMA have the highest penetration value, a desirable trait for the treatment techniques used in this study. Moreover, when we compared the application time needed for the two materials, we found that ICON was applied for a longer period of time than Helioseal F. This may have led to better penetration values. Finally, the use of hydrochloric acid as an etchant has been shown to be extremely erosive in comparison to $37 \%$ phosphoric acid gel when applied, according to the manufacturer instruction, for 120 seconds. ${ }^{22}$ Therefore, this erosion may have resulted in deeper penetration of ICON than Helioseal F.

Soviero et al. ${ }^{23}$ claimed that a one-point measurement could represent the penetration ability of an infiltrant. In our study, penetration was assessed using the mean value of multiple points rather than one point to get a clearer idea of the materials' penetration capacity. Lausch et al. ${ }^{24}$ suggested in 2015 that both a one-point measurement and the mean value of multiple measurements have similar outcomes.

\section{Microleakage}

In this study, thermocycling was used to create an artificial aging process that would permit the evaluation of restorative material deterioration. ${ }^{25}$ The thermal expansion and contraction between the sealing material and the enamel create stress, which leads to the material's failure. ${ }^{26}$ Hot water can also accelerate the hydrolysis between the enamel and the restorative material. ${ }^{27}$ Thermocycling of 1,000 cycles $\left(5-55^{\circ} \mathrm{C}\right)$ was selected for this study to simulate aging that resembles oral environment. ${ }^{27}$

Microleakage is an essential factor when assessing the sealing ability of different materials. Hence, we presume that these microleakage results may indicate future clinical outcomes. $\mathrm{AgNO}_{3}$ was selected because of its extremely small ions $(0-0.59 \mathrm{~nm})$, which are even smaller than ordinary oral bacteria $(0.5-1 \mu \mathrm{m}){ }^{28}$ Thus, any material that stops microleakage of the $\mathrm{AgNO}_{3}$ ion should also stop microleakage of bacteria and its byproducts.

There is no standardized laboratory technique to evaluate the microleakage of fissure sealants, which makes it difficult to compare our results with other studies. We used a quantitative approach to analyze our data by dividing the total $\mathrm{AgNO}_{3}$ volume by the total volume of our area of interest (the fissure area). The resulting ratio provides a $3 \mathrm{D}$ representation of the microleakage that occurred.

Our study outcomes revealed that ICON results in significantly less microleakage than Helioseal $F$ when applied on noncavitated white spot lesions in pit and fissure of permanent teeth. This can be explained by the higher penetration ability of the material and the coefficient of thermal contraction and expansion that resemble the tooth substrate.

Few studies have reported that resin infiltrant could be used to prevent microleakage in the margins of restorations ${ }^{29,30}$ or used as a replacement for the adhesive used to restore a superficial enamel lesion. ${ }^{31}$ However, one recent clinical study used resin infiltrant solely as a sealing material to stop the progression of a noncavitated pit-and-fissure enamel lesion. That study found that ICON effectively prevented the progression of caries. ${ }^{32}$ These studies support our results.

The success of resin infiltrant in the early noncavitated lesions can be an advantage in treating pediatric patients since it limits the need for teeth drilling. In addition, it is a short and painless procedure that does not require anesthesia that can be easily accepted by young children.

Further longitudinal in vivo studies are recommended to investigate the durability and cost-effectiveness of ICON when used in the fissures of teeth.

\section{Conclusion}

Under our study conditions, we can conclude that the resin infiltrant ICON has significantly more penetration and less microleakage values than Helioseal $\mathrm{F}$ when used in initial noncavitated carious fissures on permanent teeth.

\section{Clinical Significance}

As retention of pit and fissure sealant poses a challenge in preventing dental caries; resin infiltrant showed superior retention and can provide a good alternative for adhesives and fissure sealants.

\section{Acknowledgment}

The authors acknowledge with thanks the Deanship of Scientific Research (DSR) and Advanced Technology Dental Research Lab at King Abdulaziz University, Jeddah, Kingdom of Saudi Arabia for technical support.

\section{References}

1. Dye BA, Tan S, Smith V, et al. Trends in oral health status: united States, 1988-1994 and 1999-2004. Vital and health statistics. series 11. Data Natl health Surv 2007(248):1-92.

2. US Department of Health and Human Services, Oral health in America: a report of the surgeon general. Rockville, MD: US Department of Health and Human Services, National Institute of Dental and Craniofacial Research, National Institutes of Health; 2000. NIH Publication no. 00-4713 2014. Available from: https://profiles.nlm. nih.gov/ps/access/NNBBJT.pdf.

3. Khan SQ, Khan NB, ArRejaie AS. Dental caries. A meta-analysis on a Saudi population. Saudi Med J 2013;34(7):744-749.

4. Al Agili DE. A systematic review of population-based dental caries studies among children in Saudi Arabia. Saudi Dent J 2013;25(1):3-11. DOI: 10.1016/j.sdentj.2012.10.002.

5. Marthaler TM. Changes in dental caries 1953-2003. Caries Res 2004;38(3):173-181. DOI: 10.1159/000077752.

6. Mertz-Fairhurst EJ, Curtis JW, Ergle JW, et al. Ultraconservative and cariostatic sealed restorations: results at year 10. J Am Dent Assoc 1998;129(1):55-66. DOI: 10.14219/jada.archive.1998.0022.

7. Simonsen RJ. Sealants and caries. J Am Dent Assoc 2013;144(7): 767-768. DOI: 10.14219/jada.archive.2013.0182.

8. Erdemir U, Sancakli HS, Yaman BC, et al. Clinical comparison of a flowable composite and fissure sealant: a 24-month split-mouth, 
randomized, and controlled study. J Dent 2014;42(2):149-157. DOI: 10.1016/j.jdent.2013.11.015.

9. Jodkowska E. Efficacy of pit and fissure sealing: long-term clinical observations. Quintessence Int 2008;39(7):593-602.

10. Paris S, Lausch J, Selje T, et al. Comparison of sealant and infiltrant penetration into pit and fissure caries lesions in vitro. J Dent 2014;42(4):432-438. DOI: 10.1016/j.jdent.2014.01.006.

11. Arslan S, Lipski L, Dubbs K, et al. Effects of different resin sealing therapies on nanoleakage within artificial non-cavitated enamel lesions. Dent Mater J 2018;37(6):981-987. DOI: 10.4012/dmj. 2017-027.

12. Corry A, Millett D, Creanor S, et al. Effect of fluoride exposure on cariostatic potential of orthodontic bonding agents: an in vitro evaluation. J Orthod 2003;30(4):323-329. DOI: 10.1093/ortho/ 30.4.323.

13. Ismail A, Sohn W, Tellez M, et al. The international caries detection and assessment system (ICDAS): an integrated system for measuring dental caries. Community Dent Oral Epidemiol 2007;35(3):170-178. DOI: 10.1111/j.1600-0528.2007.00347.x.

14. Paris $\mathrm{S}$, Bitter $\mathrm{K}$, Renz $\mathrm{H}$, et al. Validation of two dual fluorescence techniques for confocal microscopic visualization of resin penetration into enamel caries lesions. Microsc Res Tech 2009;72(7):489-494. DOI: 10.1002/jemt.20701.

15. Hiremath S. Textbook of preventive and community dentistry. India: Elsevier; 2011.

16. Baygin O, Korkmaz FM, Tüzüner T, et al. The effect of different enamel surface treatments on the microleakage of fissure sealants. Lasers Med Sci 2012;27(1):153-160. DOI: 10.1007/s10103-0110918-x.

17. Simonsen R. A review of the clinical application and performance of pit and fissure sealants. Aust Dent J 2011;56:45-58. DOI: 10.1111/j.18347819.2010.01295.x.

18. Theuns H, Van Dijk J, Jongebloed W, et al. The mineral content of human enamel studied by polarizing microscopy, microradiography and scanning electron microscopy. Arch Oral Biol 1983;28(9):797-803. DOI: 10.1016/0003-9969(83)90035-3.

19. Wilson PR, Beynon AD. Mineralization differences between human deciduous and permanent enamel measured by quantitative microradiography. Arch Oral Biol 1989;34(2):85-88. DOI: 10.1016/00039969(89)90130-1.

20. Fan P, Seluk L, O'brien W. Penetrativity of sealants: I. J Dent Res 1975;54(2):262-264.
21. Sideridou I, Tserki V, Papanastasiou G. Study of water sorption, solubility and modulus of elasticity of light-cured dimethacrylatebased dental resins. Biomaterials 2003;24(4):655-665. DOI: 10.1016/ S0142-9612(02)00380-0.

22. Meyer-Lueckel H, Paris S, Kielbassa AM. Surface layer erosion of natural caries lesions with phosphoric and hydrochloric acid gels in preparation for resin infiltration. Caries Res 2007;41:223-230. DOI: 10.1159/000099323.

23. Soviero $V$, Paris $S$, Leal $S$, et al. Ex vivo evaluation of caries infiltration after different application times in primary molars. Caries Res 2013;47(2):110-116. DOI: 10.1159/000345065.

24. Lausch J, Paris S, Selje T, et al. Resin infiltration of fissure caries with various techniques of pretreatment in vitro. Caries Res 2015;49(1): 50-55. DOI: 10.1159/000366082. Available from: https://www.karger. com/Article/Abstract/366082.

25. Li H, Burrow MF, Tyas MJ. The effect of thermocycling regimens on the nanoleakage of dentin bonding systems. Dent Mater 2002;18(3): 189-196. DOI: 10.1016/S0109-5641(01)00081-1.

26. Asaka Y, Amano S, Rikuta A, et al. Influence of thermal cycling on dentin bond strengths of single-step self-etch adhesive systems. Oper Dent 2007;32(1):73-78. DOI: 10.2341/06-21.

27. De Munck J, Van Landuyt K, Coutinho E, et al. Micro-tensile bond strength of adhesives bonded to class-I cavity-bottom dentin after thermo-cycling. Dent Mater 2005;21(11):999-1007. DOI: 10.1016/ j.dental.2004.11.005

28. Douglas W, Fields R, Fundingsland J. A comparison between the microleakage of direct and indirect composite restorative systems. J Dent 1989;17(4):184-188. DOI: 10.1016/0300-5712(89)90072-9.

29. Tulunoglu $O$, Tulunoglu I, Antonson S, et al. Effectiveness of an infiltrant on sealing of composite restoration margins with/without artificial caries. J Contemp Dent Pract 2014;15(6):717-725. DOI: 10.5005/jp-journals-10024-1605.

30. Espigares J, Hayashi J, Shimada Y, et al. Enamel margins resealing by low-viscosity resin infiltration. Dent Mater J 2018;37(2):350-357. DOI: 10.4012/dmj.2017-153.

31. Kielbassa AM, Ulrich I, Schmidl R, et al. Resin infiltration of deproteinised natural occlusal subsurface lesions improves initial quality of fissure sealing. Int J Oral Sci 2017;9(2):117. DOI: 10.1038/ijos.2017.15.

32. Anauate-Netto $C$, Neto $B L$, Amore $R$, et al. Caries progression in non-cavitated fissures after infiltrant application: a 3-year follow-up of a randomized controlled clinical trial. J Appl Oral Sci 2017;25(4): 442-454. DOI: 10.1590/1678-7757-2016-0633. 